\title{
Profiling of Target Molecules for Immunotherapy in Mesenchymal Tumors
}

\author{
Takuma Hayashi a, b, k, Tomoyuki Ichimurac, Kenji Sano ${ }^{\mathrm{d}}$, Susumu Tonegawa ${ }^{\mathrm{e}}$, \\ Yae Kanaif, g, Dorit Zharharyb, h, Hiroyuki Aburatani ${ }^{i}$, \\ Nobuo Yaegashi ${ }^{j}$, Ikuo Konishi ${ }^{\mathrm{a}}$
}

\section{To the Editor}

The use of anti-human signal molecules monoclonal antibodies (mAbs) for malignant tumors therapy has achieved considerable success in recent years. Antibody drug conjugates are powerful new clinical treatment options for lymphomas and solid tumors, and immunomodulatory antibodies have also recently achieved remarkable clinical success. The development of therapeutic antibodies requires a deep understanding of malignant tumor serology, protein-engineering techniques, mechanisms of action and resistance, and the interplay between the immune system and tumorigenesis. This review outlines the fundamental strategies, which are required to establish antibody therapies for patients with mesenchymal tumors through iterative approaches to target and antibody selection, extending from preclinical studies to human trials.

A type of drug blocks certain proteins made by some types of immune system cells, such as T cells and some malignant tumor cells. These proteins help keep immune responses in check, and can keep T cells from killing malignant tumor cells. When these proteins are blocked, the "brakes" on the immune

Manuscript submitted May 21, 2019, accepted May 27, 2019

aNational Hospital Organization Kyoto Medical Center, Kyoto, Japan bSIGMA-Aldrich Collaboration Lab and Promoting Business using Advanced Technology, Japan Science and Technology Agency (JST), Tokyo, Japan ${ }^{\circ}$ Department of Obstetrics and Gynecology, Osaka City University Graduate School of Medicine, Osaka, Japan

dPathology Division, Iida City Hospital, Iida, Japan

ePicower Institute, Massachusetts Institute of Technology, Cambridge, MA, USA

fPathology Division, Keio University School of Medicine, Tokyo, Japan

'The International Human Epigenome Consortium (IHEC) and CREST, Japan Science and Technology Agency (JST), Tokyo, Japan

hSIGMA-Aldrich Israel, Jerusalem, Israel

${ }^{i}$ The Cancer System Laboratory, Research Center for Advanced Science and Technology, The University of Tokyo, Tokyo, Japan

${ }^{j}$ Department of Obstetrics and Gynecology, Tohoku University Graduate School of Medicine, Sendai, Japan

${ }^{\mathrm{k}}$ Corresponding Author: Takuma Hayashi, Cancer Medical Laboratory, National Hospital Organization Kyoto Medical Center, 1-1, Fukabuka mukaihata-machi, Fushimi-ku, Kyoto-city, Kyoto 612-8555, Japan. Email: yoyoyo@hotmail.com

doi: https://doi.org/10.14740/jocmr3886 system are released and T cells are able to kill malignant tumor cells better. Examples of checkpoint proteins found on T cells or malignant tumor cells include programmed death 1 (PD1)/programmed death ligand 1 (PD-L1) and cytotoxic T-lymphocyte-associated protein 4 (CTLA-4)/B7-homolog-1(B7-1)/ B7-homolog-2(B7-2) (Fig. 1). A significant inverse correlation between PD-L1 expression and intraepithelial CD8-positive Tlymphocyte count suggested that PD-L1 on tumor cells may suppress anti-tumor CD8-positive T cells, also called as cytotoxic T cells [1]. Some immune checkpoint inhibitors (ICIs) are used to treat malignant tumors. The probability of response to ICIs is closely related to the nature of tumor's immune microenvironment. For instance, non-small cell lung carcinoma has emerged as the prototypical tumor types that are responsive to ICIs, and also has PD-L1 immunohistochemical (IHC) companion diagnostic biomarker, which is used to determine whether pembrolizumab or nivolumab, which are anti-human PD-1 monoclonal immunoglobulin (Ig) G4 antibodies, monotherapy will be given $[2,3]$. In contrast, the immune microenvironment of mesenchymal tumors is still poorly understood, and biomarkers predictive of immunotherapy response are greatly needed to establish anti-mesenchymal tumors therapy by ICIs.

Diagnoses for mesenchymal tumors are rare and published case series typically lump heterogeneous mesenchymal tumors together. Due to this and because of the use of various different PD-L1 antibodies in different studies, the reported incidence of PD-L1 positivity in mesenchymal tumors has varied greatly in the literature, ranging from $0 \%$ to $60 \%$ [4-13]. In mesenchymal tumors, high expression of PD-L1 mRNA is associated with shorter metastasis-free survival [14]. In two meta-analyses, the expression of PD-L1 was found to be a poor prognosticator in mesenchymal tumors [15].

ICIs have yielded mixed results in sarcoma. Ipilimumab, which binds to a substance called CTLA-4, had no activity in six patients with synovial sarcoma [16]. Ben-Ami reported that none of 12 patients with uterine leiomyosarcoma responded to nivolumab, which is anti-human PD-1 fully human immunoglobulin (Ig) G4 mAb [17]. Two of four patients with alveolar soft part sarcoma were reported to have partial response to anti-PD-L1 therapy [18]. A microsatellite instability (MSI), low tumor mutation burden (TMB) chondrosarcoma with $1 \%$ PD-L1 positivity, was reported to respond to nivolumab [19]. Finally, the SARC028 clinical trial reported that $18 \%$ of pa- 


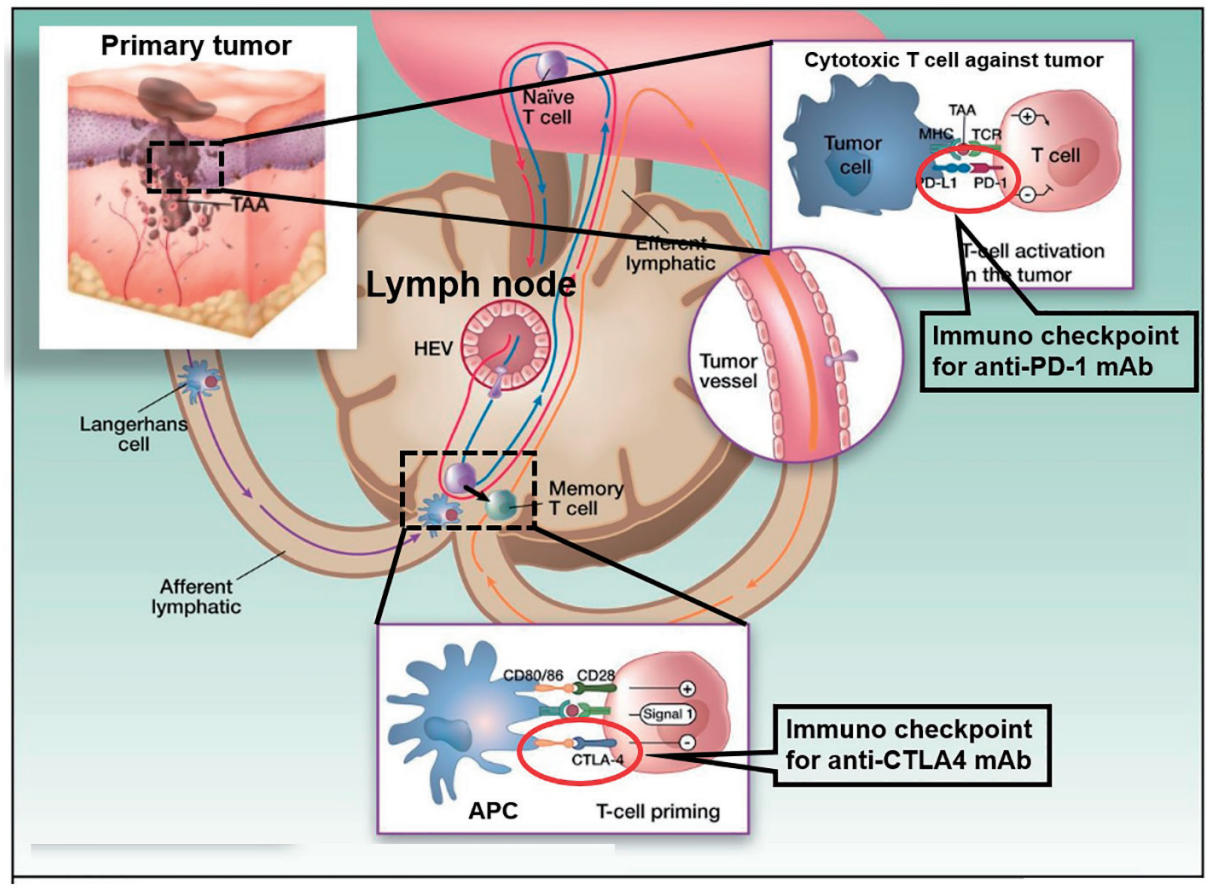

Figure 1. Cancer therapy by inhibition of negative immune regulation by programmed death 1 (PD-1) and cytotoxic T-lymphocyte-associated protein 4 (CTLA-4). The figure shows a primary melanoma and tumor-associated antigens (TAAs) that are taken up by an antigen presenting cell (APC: a Langerhans cell; left). APCs then migrate via afferent lymphatics to the sentinel skindraining lymph node, where they present TAA to naive T cells. These naive T cells continuously screen the lymphoid organs, extravasating through high endothelial venule (HEV) until their corresponding peptide is presented to them in the context of the major histocompatibility complex (MHC). When a TAA is presented in the T-cell-dependent area of a lymph node to its specific naive $T$ cell (center), the latter requires signal 1 (antigen presentation to the T-cell receptor) and 2 (costimulation) for full activation. It will then proliferate and acquire "antigen memory" and a distinct and different set of adhesion molecules that will allow it to navigate outside of the blood vessels and the lymphoid organs, to the peripheral tissues and organs, like the melanoma metastases where it will be able to be reactivated upon re-presentation of the same TAA. However, in the lymph node, T-cell activation is interrupted when signal 3, mediated through interaction between CTLA-4 and CD80/86, takes over CD28 and CD80/86 interaction. This occurs $24-48 \mathrm{~h}$ after the initiation of T-cell priming. When the memory $\mathrm{T}$ cell is recruited to the melanoma metastasis (right) and activated upon TAA re-presentation, the effector activation is decreased when PD-1 is engaged with its ligand PD-L1 that can be expressed on the tumor cell constitutively or in the context of inflammation. The figure adapted from the C. Robert and C. Verjat figure collection, Institute Gustave Roussy (Villejuif-Paris Sud, Paris, France) is modified.

tients with mesenchymal tumors had an objective response to pembrolizumab. Twenty percent of patients with liposarcoma, $10 \%$ with synovial sarcoma and $5 \%$ with bone sarcoma had a response. No response was seen in patients with leiomyosarcoma or Ewing sarcoma. Importantly, four of 10 patients with undifferentiated mesenchymal tumors had a response and two of these four patients had at least $1 \%$ neoplastic cell PD-L1 positivity. Tumors from all six pembrolizumab unresponsive patients with mesenchymal tumors were PD-L1 negative [9]. High RNA expression of B7-homolog 3 (B7-H3), transforming growth factor beta-1 (TGFB1) and T-cell immunoglobulin and mucin domains-containing protein 3 (TIM3), which are putative immunotherapy targets, has been described in sarcoma, specifically in dedifferentiated liposarcoma, undifferentiated pleomorphic sarcoma and myxofibrosarcoma [20].

Most of the immune-therapeutic focus in mesenchymal tumor types has centered on the PD-1 axis [4, 5, 10-15]. The expression of a few alternate immune-therapeutic targets, such as B7-H3, TGFB1 and TIM3, has been previously described in some mesenchymal tumor types [20,21]. Pathological studies with anti-PD-1 and/or anti-PD-L1 antibodies have yielded inconsistent findings in mesenchymal tumors partially, because of the inclusion of heterogeneous sarcoma entities in many of the relevant studies [4-12]. Using various criteria and antibodies, most studies show that $40-82 \%$ of some mesenchymal tumor types have expression of PD-L1 [4, 11, 12]. The population of mesenchymal tumors had reportedly PD-L1 positivity in $38 \%$ of cases, substantially more than the non-mesenchymal tumor population which was PD-L1 positive in $19 \%$ of cases. The expression of PD-L1 mRNA in mesenchymal tumors was not significantly different from non-mesenchymal tumor cases and was significantly under expressed in non-mesenchymal tumors. However, PD-L2 emerged as significantly overexpressed in mesenchymal tumors. PD-L2 expresses in both neoplastic and non-neoplastic cells in multiple neoplasms and its expression correlates with pembrolizumab response in head and neck squamous cell carcinoma independent of PDL1 expression [22]. Along with the high positivity PD-L1 in mesenchymal tumors, the overexpression of PD-L2 in mesenchymal tumors suggests that the communication of PD-1/ 


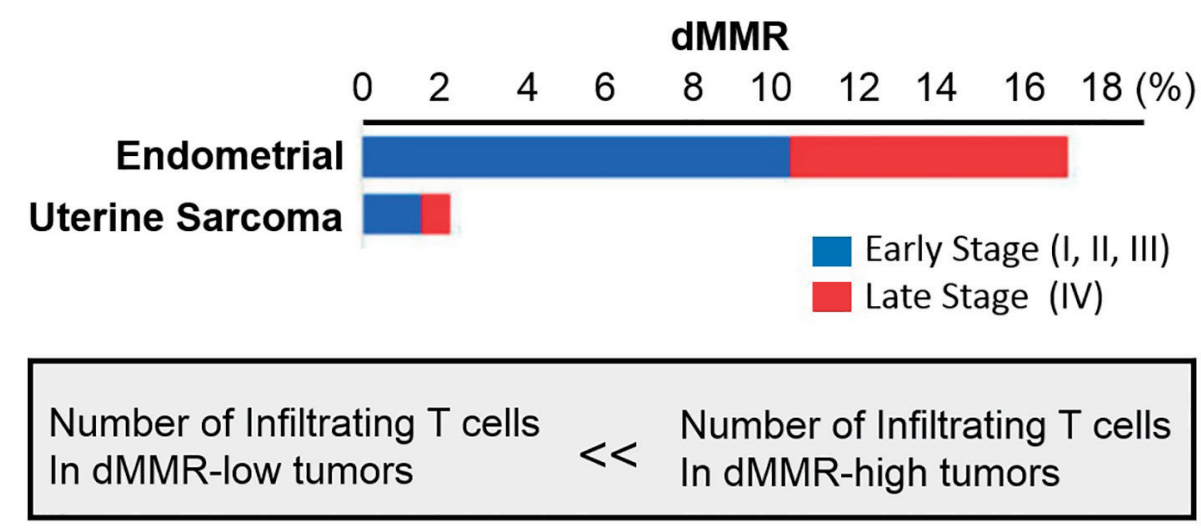

Figure 2. Mismatch repair deficiency across tumors. Proportion of tumors deficient in mismatch repair (dMMR) in each malignant tumor subtype, expressed as a percentage. Mismatch repair deficient tumors were identified in tumor subtypes tested, more often in early stage (defined as stage < IV) disease. The figure adapted from reference [8].

PD-L1/PD-L2 axis may be a key role of protective mechanism against host immune response in mesenchymal tumors. In turn, this raises the possibility that agents targeting PD-L1 may not be as therapeutically efficacious as agents targeting the PD-1 in mesenchymal tumors, and provides a possible reason for the high response rate of mesenchymal tumors to pembrolizumab [9]. As additional putative immunotherapy targets, B7-H3 and TGFB1 were remarkable in that they were both overexpressed in mesenchymal tumors and non-mesenchymal tumors. B7-H3 is immunohistochemically present in most osteosarcomas, and is associated with a poor prognosis and with poor infiltration by CD8-positive T cells [23]. Our knowledge of its functional significance in other sarcomas is limited, but its known immune-suppressive function and relatively high expression in both mesenchymal tumors and non-mesenchymal tumors suggests that it may be an important immune-suppressor in mesenchymal tumors. Overexpression of TGFB1 as the immune-suppressive cytokine has been described in mesenchymal tumors [20, 21]. Importantly, TGFB1 physiologically induces epithelial to mesenchymal transition (EMT) [24]. The elevated expression of multiple EMT-related factors also induces the process of protection from host immune-system, and these factors are exploitable as immunotherapy targets. Colony-stimulating factor 1 receptor (CSF1R) is well known to associate with macrophages and there is preclinical evidence that its blockade increases CD8-positive T-cell motility and tumor infiltration [25]. The significantly differential expression of TIM3 is observed in mesenchymal tumors or non-mesenchymal tumors. This is in conflict with the previously described overexpression of TIM3 in the Cancer Genome Atlas (TCGA) sarcoma cohort [20]. The reasons for this discrepancy are unclear, although the differences in the specific subtypes of mesenchymal tumors included in ours and TCGA cohort may be an explanation. Notably, other potential immunotherapy targets such as CTLA4 and PD-1 were either under expressed or had no expression difference in mesenchymal tumors and non-mesenchymal tumors. Functional analysis in a responding patient demonstrated rapid in vivo expansion of neo-antigen-specific T-cell clones that were reactive to mutant neo-peptides found in the tumor. These data support the hypothesis that the large proportion of mutant neo-antigens in mismatch repair-deficient (dMMR) makes them sensitive to immune checkpoint blockade, regardless of the malignant tumor tissues of origin. The surging interest in cancer immunotherapy, particularly checkpoint blockade, has further led to a focus on dMMR-positive tumors, which are notable for their substantial CD8-positive T-cell infiltrate [26]. Recent report demonstrated that uterine sarcomas were mismatch repair-deficient [9, 26-28]. Uterine sarcoma, $1.4 \%$ of stage I to stage III malignant tumors and $0.6 \%$ of stage IV malignant tumors were classified in dMMR-positive tumors [8] (Fig. 2). From these research findings, in uterine sarcoma, numbers of infiltrating CD8-positive T cells are considered to be small. Therefore, as previously reported, the anti-tumor effect of therapy by ICIs on uterine sarcoma is considered to be low.

The causal relationship between metastatic status and presence of CD8 is currently unclear, although there is preclinical evidence indicating that depletion of cytotoxic CD8-positive T cells plays an important role in the proliferation of metastatic neoplastic cells [27]. The recent research findings suggest that inflammatory status can be used to categorize sarcomas and, possibly, their clinical behavior. Further clinical studies will elucidate whether this type of profiling is also predictive of immunotherapy response. The sample size is small and we were only able to include a smattering of various sarcomas in the mesenchymal tumor category. Further studies will need large numbers of each specific sarcoma histology. Finally, histopathological studies and functional or clinical studies are needed to confirm our findings.

In conclusion, if mesenchymal tumors in particular have a very high rate of positive expression of PD-L1 mRNA and high expression of PD-L2 mRNA, which suggests a mechanism of immune-suppression, therefore uterine sarcoma in mesenchymal tumors types has possibly a high response rate to ICIs. The expression profiling of proteins demonstrated the expression of PD-L1, and positive dMMR in uterine sarcoma [9, 26-29]. Unfortunately in uterine sarcoma, late of dMMR reportedly is low, and does not have high response to ICIs [8, 29]. The clinical evidences of lymphocytes infiltration and negative signalligands such like PD-L1 in mesenchymal tumors suggest that 
the tumor microenvironment plays an important role of antitumor by ICIs.

\section{Acknowledgments}

We sincerely thank Professor Luc Van Kaer (Vanderbilt University Medical Center) for donation of LMP2-deficiency mouse.

\section{Financial Disclosure}

This study was supported in part by grants from the Japan Ministry of Education, Culture, Science and Technology (grants \#15K10709 and \#24592510), The Foundation of Osaka Cancer Research, The Ichiro Kanehara Foundation for the Promotion of Medical Science and Medical Care, The Foundation for the Promotion of Cancer Research, The Kanzawa Medical Research Foundation, The Shinshu Medical Foundation and The Takeda Foundation for Medical Science.

\section{Conflict of Interest}

All authors report no conflict of interest.

\section{Informed Consent}

Not applicable.

\section{Author Contributions}

TH: execution of research and experimental design. TI: execution of research and experimental design. KS: execution of research. ST: research cooperation. YK: execution of research and research cooperation. DZ: joint experiment and execution of research, HA: joint experiment and execution of research. NY: Joint experiment, execution of research and research supervision. KI: research supervision.

\section{References}

1. Qian X, Agematsu K, Freeman GJ, Tagawa Y, Sugane K, Hayashi T. The ICOS-ligand B7-H2, expressed on human type II alveolar epithelial cells, plays a role in the pulmonary host defense system. Eur J Immunol. 2006;36(4):906-918.

2. Chen DS, Mellman I. Oncology meets immunology: the cancer-immunity cycle. Immunity. 2013;39(1):1-10.

3. Tang J, Shalabi A, Hubbard-Lucey VM. Comprehensive analysis of the clinical immuno-oncology landscape. Ann Oncol. 2018;29(1):84-91.

4. Kim JR, Moon YJ, Kwon KS, Bae JS, Wagle S, Kim KM, Park HS, et al. Tumor infiltrating PD1-positive lympho- cytes and the expression of PD-L1 predict poor prognosis of soft tissue sarcomas. PLoS One. 2013;8(12):e82870.

5. D'Angelo SP, Shoushtari AN, Agaram NP, Kuk D, Qin LX, Carvajal RD, Dickson MA, et al. Prevalence of tumor-infiltrating lymphocytes and PD-L1 expression in the soft tissue sarcoma microenvironment. Hum Pathol. 2015;46(3):357-365.

6. Kim C, Kim EK, Jung H, Chon HJ, Han JW, Shin KH, $\mathrm{Hu} \mathrm{H}$, et al. Prognostic implications of PD-L1 expression in patients with soft tissue sarcoma. BMC Cancer. 2016;16:434.

7. Duruisseaux M, Rouquette I, Adam J, Cortot A, Cazes A, Gibault L, Damotte D, et al. [Efficacy of PD-1/PD-L1 immune checkpoint inhibitors and PD-L1 testing in thoracic cancers]. Ann Pathol. 2017;37(1):61-78.

8. Le DT, Durham JN, Smith KN, Wang H, Bartlett BR, Aulakh LK, Lu S, et al. Mismatch repair deficiency predicts response of solid tumors to PD-1 blockade. Science. 2017;357(6349):409-413.

9. Tawbi HA, Burgess M, Bolejack V, Van Tine BA, Schuetze SM, Hu J, D'Angelo S, et al. Pembrolizumab in advanced soft-tissue sarcoma and bone sarcoma (SARC028): a multicentre, two-cohort, single-arm, open-label, phase 2 trial. Lancet Oncol. 2017;18(11):1493-1501.

10. Wisdom AJ, Mowery YM, Riedel RF, Kirsch DG. Rationale and emerging strategies for immune checkpoint blockade in soft tissue sarcoma. Cancer. 2018;124(19):38193829.

11. Torabi A, Amaya CN, Wians FH, Jr., Bryan BA. PD-1 and PD-L1 expression in bone and soft tissue sarcomas. Pathology. 2017;49(5):506-513.

12. Pollack SM, He Q, Yearley JH, Emerson R, Vignali M, Zhang Y, Redman MW, et al. T-cell infiltration and clonality correlate with programmed cell death protein 1 and programmed death-ligand 1 expression in patients with soft tissue sarcomas. Cancer. 2017;123(17):3291-3304.

13. Hayashi T, Faustman DL. Development of spontaneous uterine tumors in low molecular mass polypeptide-2 knockout mice. Cancer Res. 2002;62(1):24-27.

14. Bertucci F, Finetti P, Perrot D, et al. PDL1 expression is a poor-prognosis factor in soft-tissue sarcomas. Oncoimmunology. 2017;6:1-11.

15. Zhu Z, Jin Z, Zhang M, Tang Y, Yang G, Yuan X, Yao J, et al. Prognostic value of programmed death-ligand 1 in sarcoma: a meta-analysis. Oncotarget. 2017;8(35):5957059580.

16. Maki RG, Jungbluth AA, Gnjatic S, Schwartz GK, D'Adamo DR, Keohan ML, Wagner MJ, et al. A pilot study of Anti-CTLA4 antibody ipilimumab in patients with synovial sarcoma. Sarcoma. 2013;2013:168145.

17. Ben-Ami E, Barysauskas CM, Solomon S, Tahlil K, Malley R, Hohos M, Polson K, et al. Immunotherapy with single agent nivolumab for advanced leiomyosarcoma of the uterus: Results of a phase 2 study. Cancer. 2017;123(17):3285-3290.

18. Groisberg R, Hong DS, Behrang A, Hess K, Janku F, Piha-Paul S, Naing A, et al. Characteristics and outcomes of patients with advanced sarcoma enrolled in early phase immunotherapy trials. J Immunother Cancer. 
2017;5(1):100.

19. Wagner MJ, Ricciotti RW, Mantilla J, Loggers ET, Pollack SM, Cranmer LD. Response to PD1 inhibition in conventional chondrosarcoma. J Immunother Cancer. 2018;6(1):94.

20. The Cancer Genome Atlas Research Network. Comprehensive and integrated genomic characterization of adult soft tissue sarcomas. Cell. 2017;171(4):950-965, e28.

21. Hayashi T, Kawano M, Ichimura T, Ida K, Ando H, Zharhary D, Kanai Y, et al. Molecular pathology and novel clinical therapy for uterine leiomyosarcoma. Anticancer Res. 2016;36(10):4997-5007.

22. Yearley JH, Gibson C, Yu N, Moon C, Murphy E, Juco J, Lunceford J, et al. PD-L2 Expression in human tumors: relevance to Anti-PD-1 therapy in cancer. Clin Cancer Res. 2017;23(12):3158-3167.

23. Li G, Quan Y, Che F, Wang L. B7-H3 in tumors: friend or foe for tumor immunity? Cancer Chemother Pharmacol. 2018;81(2):245-253.

24. Kalluri R, Weinberg RA. The basics of epithelial-mesen- chymal transition. J Clin Invest. 2009;119(6):1420-1428.

25. Peranzoni E, Lemoine J, Vimeux L, Feuillet V, Barrin S, Kantari-Mimoun C, Bercovici N, et al. Macrophages impede CD8 T cells from reaching tumor cells and limit the efficacy of anti-PD-1 treatment. Proc Natl Acad Sci U S A. 2018;115(17):E4041-E4050.

26. Quiroga D, Lyerly HK, Morse MA. Deficient mismatch repair and the role of immunotherapy in metastatic colorectal cancer. Curr Treat Options Oncol. 2016;17(8):41.

27. Seledtsov VI, Goncharov AG, Seledtsova GV. Clinically feasible approaches to potentiating cancer cellbased immunotherapies. Hum Vaccin Immunother. 2015;11(4):851-869.

28. Le DT, Uram JN, Wang H, Bartlett BR, Kemberling $\mathrm{H}$, Eyring AD, Skora AD, et al. PD-1 blockade in tumors with mismatch-repair deficiency. N Engl J Med. 2015;372(26):2509-2520.

29. Lee V, Murphy A, Le DT, Diaz LA, Jr. Mismatch repair deficiency and response to immune checkpoint blockade. Oncologist. 2016;21(10):1200-1211. 